\title{
Key Themes in Recent Research on Interpersonal Functioning in Borderline Personality Disorder
}

\author{
John Michael* \\ Division of Psychology, Faculty of Natural Sciences \\ University of Stirling \\ FK9 4LA \\ Stirling, Scotland, UK
}

Simon van Baal

Philosophy Department

Monash University

VIC 3800

Melbourne, Australia

* Corresponding Author:

Email: john.michael@stir.ac.uk

Tel: +447452839497 


\begin{abstract}
Purpose of Review: We provide an overview of new ideas and directions of research on interpersonal functioning in borderline personality disorder (BPD).

Recent Findings: We highlight the following key themes emerging in recent and ongoing research: investigation of the cognitive and motivational mechanisms which underpin impairments of interpersonal functioning in BPD; tracking BPD across the lifespan with earlier interventions and longitudinal studies; expansion of the scope of interest to family members of individuals with BPD and to people in the general population with levels of BPD traits; and investigating BPD online, not only by using internet-based testing platforms, but also by studying the social media use of individuals with BPD.
\end{abstract}

Summary: The concise discussion of recent research on BPD provided here, together with the identification of key themes emerging from this work, provide a snapshot of ongoing work devoted to better understanding interpersonal functioning in borderline personality disorder.

Keywords: borderline personality disorder, trust, commitment, early intervention, social media

\title{
Introduction
}

Borderline personality disorder (BPD) is a well-established psychiatric diagnosis associated with significant psychosocial impairments, and considerable economic costs due to intensive use of treatment and loss of productivity ${ }^{1}$. The disorder has biological and developmental roots and a lifetime prevalence of $2 \%-6 \%{ }^{2}$. It is marked by conflicted relationships, difficulty trusting other people, fear of abandonment, and patterns of overinvolvement/withdrawal as well as idealization/devaluation of relationships. In more general terms, impairment in interpersonal functioning has been highlighted as a core feature of psychopathology in $\mathrm{BPD}^{3}$. 
Moreover, other characteristic symptoms of BPD, such as anger, affective instability, suicidal behavior, and impulsiveness, mainly manifest within interpersonal contexts ${ }^{4}$. A key challenge for ongoing and future research is therefore to develop a better understanding of interpersonal functioning in BPD. In the present structured review, we highlight the following key themes that have emerged in this research: investigation of the cognitive and motivational mechanisms which underpin impairments of interpersonal functioning in BPD; tracking BPD across the lifespan with earlier interventions and longitudinal studies; expansion of the scope of interest to family members of individuals with BPD and to people in the general population with levels of BPD traits; and investigation of BPD online, not only by using internet-based testing platforms, but also by studying the social media use of individuals with BPD.

\section{Zooming in on Mechanisms}

In order to develop effective treatments for BPD, and to understand its causes, it is crucially important to obtain scientific knowledge of the cognitive and motivational mechanisms which underpin it. To this end, controlled experimentation is needed in order to identify differences in interpersonal functioning between individuals with BPD and controls, and to test hypotheses about the causes of these differences.

\subsection{Triggering Symptoms}

One challenge in this regard is that individuals with BPD do not always exhibit irregularities with respect to their willingness to trust others or in their approach to social interactions and relationships. Rather, these symptoms are exhibited only when triggered, for example when social expectations are disappointed or fears of abandonment are elicited. Moreover, it is also noteworthy that irregularities in the interpersonal functioning of patients with BPD are not always or only negative (e.g. a lack of trust), but sometimes highly positive. Rather, the characteristic pattern is one of alternation between idealization and devaluation of relationships 5 .

To gain a fuller understanding of the cognitive and motivational processes underlying the problems in interpersonal functioning experienced by patients with $\mathrm{BPD}$, it is therefore crucial to focus not only on the negative attitudes, such as mistrust, which are sometimes 
exhibited, but instead to investigate the alternations between highly positive and highly negative attitudes. What external factors trigger or exacerbate these alternations, and what internal processes drive them? To investigate this, Chennells and colleagues ${ }^{6}$ recently devised a reactivity induction to trigger either idealization or devaluation of a partner within a controlled lab setting (in two separate groups), and to measure the effects which this reactivity induction had upon participants with BPD, compared to a control group. They found that participants with a BPD diagnosis were significantly more sensitive to this manipulation. In particular, those participants with BPD who were exposed to the devaluation induction exhibited lower levels of trust and commitment in an economic game (see next section).

\subsection{Trust and Commitment: Two Sides of a Coin}

In their recent study, Chennells and colleagues homed in on trust and commitment as complementary prosocial attitudes which serve to stabilize healthy relationships. In order to maintain healthy relationships, it is important to be willing to sacrifice time, effort, money and other resources (commitment), and also to be willing to rely on others to do so as well (trust). Although trust and commitment are distinct constructs, they are highly correlated, and indeed dynamically interrelated: people tend to increase commitment towards partners who exhibit trustworthiness, and to withdraw commitment from partners who prove untrustworthy. And indeed, the alternations to extreme modes of idealization and devaluation which are characteristic of BPD may be driven in part by the dynamic interaction of commitment and trust: if a partner exhibits a level of commitment that is higher than expected, this may elicit trust, and consequently also a high level of commitment (idealization). On the other hand, if a partner exhibits a level of commitment that is lower than expected, this may trigger mistrust, and consequently elicit a withdrawal of commitment (devaluation). This builds on previous work showing enhanced sensitivity to commitment violations among individuals with high levels of BPD traits ${ }^{7}$, and also on earlier work showing difficulties in maintaining trust among individuals with $\mathrm{BPD}^{8}$.

\section{BPD Across the Lifespan}


Clinical onset of BPD usually occurs in adolescence or early adulthood, while diagnosis lags significantly ${ }^{9}$, with impairments in interpersonal functioning persisting throughout the lifespan ${ }^{10}$, and severity of symptoms in adolescence and early adulthood predicting outcomes later in life $\left.{ }^{11},{ }^{12}\right)$.

\subsection{Early Interventions}

Difficulties in interpersonal functioning early on can lead to cascading effects upon educational progress, professional development, substance abuse and other mental health problems ${ }^{13},{ }^{14},{ }^{15}$. In order to mitigate these potentially cascading effects, early interventions have recently been developed, with a focus on supporting the abilities of young people with BPD to progress in educational and professional development ${ }^{10,16,17,18 .}$ Early interventions are however not without potential risks: Kulkarni ${ }^{19}$ for example points out that early diagnosis introduces the risk of stigmatization, and indeed that the label 'BPD' may tend to invalidate the traumatic origins which are characteristic of BPD.

\subsection{Longitudinal studies}

In order to monitor the efficacy of early interventions - and indeed of any treatment - it is important to carry out longitudinal studies which track the course of BPD over the lifespan ${ }^{11,12,20}$.This research is complemented by studies investigating the developmental precursors of BPD in childhood ${ }^{21}$.

\section{Expanding the Population of Interest}

In order to better understand impairments in interpersonal functioning among individuals with $\mathrm{BPD}$, it has proven fruitful to zoom out and to expand the population of interest. More specifically, research has begun to probe interpersonal functioning among the family members of individuals with BPD, and also to investigate the interpersonal functioning of individuals in the general population with high, yet subthreshold, levels of BPD traits. This promises to shed light on the interaction between genetic and environmental factors, and also to better 
understand the role of environmental factors in giving rise to the symptoms of BPD. Moreover, this may provide insight into the magnitude of societal problems related to BPD.

\subsection{The Family Context}

One study ${ }^{22}$ found that the close relatives of individuals with BPD exhibit some signs of the difficulties in social cognition and interaction that are characteristic of individuals with BPD. This is relevant to attempts to better understand the aetiology, and hence also the treatment, of BPD. In addition, they report evidence of social-cognitive impairments in the BPD group which are consistent with previous research and thus contribute to firming up those previous findings. It must be noted however that it is not straightforward whether any potential impairments in interpersonal functioning of family members should be interpreted as evidence of a genetic component. A plausible alternative interpretation would be that living with a person with BPD can lead to such impairments - in particular among the children of individuals with BPD.

\subsection{Subthreshold BPD Traits}

It has been shown that subthreshold BPD trait levels among individuals in the general population are indeed associated with difficulties in interpersonal functioning and can have clinical significance, as well as deleterious real-world consequences ${ }^{23}$. Building on this,one study ${ }^{7}$ showed that individuals in the general population with high levels of BPD traits exhibit a heightened sensitivity to violations of everyday commitments. This has the important implication that it may be possible to study the mechanisms giving rise to impairments in interpersonal functioning in BPD by investigating members of the general population.

\section{Testing BPD Online}

Even prior to the 2020 Lockdown in response to the Covid-19 pandemic, recent decades have seen large swathes of social life shift online. This has provided new opportunities for people to seek out new forms of social interaction, and also for researchers to access new groups of participants to test in new formats. 


\subsection{Online Data Collection}

The trend towards online participant databases provides new opportunities to researchers. For example, Ooi and colleagues ${ }^{24}$ used the internet to access and test participants with subclinical levels of BPD traits. They note that, among such participant pools, the prevalence of clinically relevant symptoms such as depression, general anxiety and trauma exposure is comparable to representative samples, while the levels of social anxiety and the prevalence of unemployment and substance abuse problems are higher than in the general population. Moreover, crowdsourcing makes it possible to access a culturally and geographically broader sample.

\subsection{BPD on Social Media}

Social media constitute an increasingly central arena of social interaction for people in general. Indeed, we may expect this to be all the more true of individuals with high BPD features, given that the difficulties they typically experience with interpersonal relations may lead them to be particularly motivated to engage with social media in order to satisfy a pronounced need for social connection. In one of the few studies looking at BPD features within this domain, elevated risks of problematic Facebook use (addictive behaviors) were found in a group of adolescents and young adults exhibiting a 'borderline' profile (defined as showing a combination of high borderline personality features, depressive symptoms, social anxiety, and sensation seeking $)^{25}$. Building on this, Ooi and colleagues ${ }^{24}$ found that interpersonal difficulties that individuals with high BPD features experience are also displayed in social media behavior.

\section{Conclusion}

We have provided an overview of new ideas and directions of research on interpersonal functioning in borderline personality disorder (BPD). In particular, we have highlighted four key themes emerging in recent and ongoing research: investigation of the cognitive and motivational mechanisms which underpin impairments of interpersonal functioning in BPD; tracking BPD across the lifespan with earlier interventions and longitudinal studies; expansion of the scope of interest to family members of individuals with BPD and to people in the general population with levels of BPD traits; and investigating BPD online, not only by using internet- 
based testing platforms, but also by studying the social media use of individuals with BPD. The concise discussion of recent research on BPD provided here, together with the identification of key themes emerging from this work, provide the reader with a structured overview of ongoing work devoted to better understanding interpersonal functioning in borderline personality disorder.

\section{Key points}

○ Recently, there has been an increased focus on investigating the cognitive and motivational mechanisms which underpin impairments of interpersonal functioning in BPD.

- Researchers are attempting to track BPD across the lifespan with earlier interventions and longitudinal studies.

- There has been an expansion of the scope of interest to family members of individuals with BPD and to people in the general population with levels of BPD traits.

○ The internet opens up new opportunities for social interactions for individuals with $\mathrm{BPD}$, and also for researchers to investigate the social media use of individuals with BPD.

\section{Acknowledgements}

John Michael was supported by a a Starting Grant from the European Research Council (nr. 679092, SENSE OF COMMITMENT).

\section{References}


${ }^{1}$ Soeteman, D. I., Roijen, L. H. V., Verheul, R., \& Busschbach, J. J. The economic burden of personality disorders in mental health care. Journal of Clinical Psychiatry 69, 259 (2008).

${ }^{2}$ Chanen, A. M., \& Kaess, M. (2012). Developmental pathways to borderline personality disorder. Current psychiatry reports, 14(1), 45-53.

${ }^{3}$ Euler, S., Nolte, T., Constantinou, M., Griem, J., Montague, P. R., Fonagy, P., \& Personality and Mood Disorders Research Network. (2019). Interpersonal problems in borderline personality disorder: associations with mentalizing, emotion regulation, and impulsiveness. Journal of Personality Disorders, 1-17.

${ }^{4}$ Sharp, C., Venta, A., Vanwoerden, S., Schramm, A., Ha, C., Newlin, E., ... \& Fonagy, P. (2016). First empirical evaluation of the link between attachment, social cognition and borderline features in adolescents. Comprehensive psychiatry, 64, 4-11.

5 Domes, G., Schulze, L., \& Herpertz, S. C. (2009). Emotion recognition in borderline personality disorder-A review of the literature. Journal of personality disorders, 23(1), 6-19.

**6 Chennells M, Nolte T, Ooi J, Fonagy P, (Under Review). Trust and commitment in borderline personality disorder.

This is the first study investigating trust and commitment in a patient group, and also the first study to utilize a reactivity induction to trigger idealization and devaluation behavior.

**7 Ooi, J., Francová, A., Székely, M., \& Michael, J. (2018). The Sense of Commitment in Individuals With Borderline Personality Traits in a Non-clinical Population. Frontiers in psychiatry, 9, 519.

This was the first study investigating the sense of commitment among people with high levels of BPD traits.

${ }^{8}$ King-casas, B., Sharp, C., Lomax-bream, L., Lohrenz, T., et al. (2008). The Rupture and Repair Personality Disorder. Science, 321(8), 806-811. http://doi.org/10.1126/science. 1156902 
${ }^{9}$ Chanen, A., Sharp, C., Hoffman, P. (2017). Prevention and early intervention for borderline personality disorder: a novel public health priority. World Psychiatry, 16(2), 215.

${ }^{10}$ Chanen, A. M., Nicol, K., Betts, J. K., \& Thompson, K. N. (2020). Diagnosis and Treatment of Borderline Personality Disorder in Young People. Current Psychiatry Reports, 22(5), 2525.

${ }^{11}$ Alvarez-Tomás, I., Soler, J., Bados, A., Martín-Blanco, A., Elices, M., Carmona, C., ... \& Pascual, J. C. (2017). Long-term course of borderline personality disorder: a prospective 10year follow-up study. Journal of personality disorders, 31(5), 590-605.

${ }^{12}$ Zanarini, M. C., Temes, C. M., Frankenburg, F. R., Reich, D. B., et al (2018). Description and prediction of time-to-attainment of excellent recovery for borderline patients followed prospectively for 20 years. Psychiatry research, 262, 40-45.

${ }^{13}$ Caruana E, Allott K, Farhall J, Parrish EM, Davey CG, Chanen AM, et al. Factors associated with vocational disengagement among young people entering mental health treatment. Early Interv Psychiatry. 2018. https://doi. org/10.1111/eip.12718.

${ }^{14}$ Juurlink TT, ten Have M, Lamers F, van Marle HJF, Anema JR, de Graaf R, et al. Borderline personality symptoms and work performance: a population-based survey. BMC Psychiatry. 2018;18(1). https://doi.org/10. 1186/s12888-018-1777-9.

${ }^{15}$ Ralston K, Feng Z, Everington D, Dibben C. Do young people not in education, employment or training experience long-term occupational scarring? A longitudinal analysis over 20 years of follow-up. Contemp Soc Sci. 2016;11(2-3):203-21. https://doi.org/10.1080/21582041.2016.1194452.

${ }^{16}$ Dahl, R. E., Allen, N. B., Wilbrecht, L., \& Suleiman, A. B. (2018). Importance of investing in adolescence from a developmental science perspective. Nature, 554(7693), 441-450.

${ }^{17}$ Hegelstad WTV, ten Velden Hegelstad W, Joa I, Heitmann L, Johannessen JO, et al. Job and school prescription: a local adaptation to individual placement and support for first episode psychosis. Early Interv Psychiatry. 2018. https://doi.org/10.1111/eip.12686. 
${ }^{18}$ Sawyer SM, Azzopardi PS, Wickremarathne D, Patton GC. The age of adolescence. Lancet Child Adolesc Health. 2018;2(3):223-8.

19 Kulkarni, J. (2015): https://theconversation.com/borderline-personality-disorder-is-ahurtful-label-for-real-suffering-time-we-changed-it-41760

${ }^{20}$ Caruana E, Farhall J, Cotton SM, Parrish E, van-der-El K, Davey CG, et al. Vocational engagement among young people entering mental health treatment compared with their general population peers. Early Interv Psychiatry. 2018. https://doi.org/10.1111/eip.12712.

${ }^{21}$ Edinboro, S. R., Nolte, T., \& Vilares, I. (2018). The neurodevelopmental trajectory of Borderline Personality Disorder: a review (No. e27414v1). PeerJ Preprints.

${ }^{22}$ Ortega-Díaz, Jonatan García-Campos , José M Rico-Gomis, Carlos Cuesta-Moreno, et al. (Under Review) Social cognition and social functioning in people with borderline personality disorder and their first-degree relatives.

${ }^{23}$ Thompson KN, Jackson H, Cavelti M, Betts J, McCutcheon L, Jovev M, et al. The clinical significance of subthreshold borderline personality disorder features in outpatient youth. $J$ Personal Disord. 2018;23:1-11.

**24 Ooi, J., Michael, J., Lemola, S., Butterfill, et al. (2020). interpersonal functioning in Borderline personality Disorder traits: A Social Media perspective. Scientific Reports, 10(1), $1-8$.

This is the study investigating the behavior of people with high levels of BPD traits on social media.

${ }^{25}$ Moreau, A., Laconi, S., Delfour, M. \& Chabrol, H. Psychopathological profiles of adolescent and young adult problematic Facebook users. Computers in Human Behavior 44, 64-69 (2015). 